nephron

Experimental

Nephrology

and Genetics
Nephron 2016;133:287-295

DOI: $10.1159 / 000447717$
Received: April 23, 2015

Accepted after revision: June 1, 2016 Published online: July 29, 2016

\title{
WNK3 Kinase Enhances the Sodium Chloride Cotransporter Expression via an ERK 1/2 Signaling Pathway
}

\author{
Dexuan Wang ${ }^{\mathrm{a}, \mathrm{b}}$ Yiqian Zhang ${ }^{\mathrm{a}}$ Jinhua Han ${ }^{\mathrm{c}}$ Shufang Pan ${ }^{\mathrm{a}} \mathrm{Ning}_{\mathrm{Xu}} \mathrm{a}^{\mathrm{a}}$ \\ Xiuyan Feng $^{\mathrm{d}, \mathrm{e}}$ Zhizhi Zhuang $^{\mathrm{d}}$ Courtney Caroti ${ }^{\mathrm{d}, \mathrm{e}}$ Jieqiu Zhuang ${ }^{\mathrm{a}}$ \\ Robert S. Hoover ${ }^{d, e}$ Dingying Gu ${ }^{a}$ Qiyi Zeng ${ }^{b}$ Hui Cai ${ }^{d, e}$ \\ ${ }^{a}$ Department of Nephrology, The Second Affiliated Hospital, Wenzhou Medical University, Wenzhou, Zhejiang, \\ bPediatric Center of Zhujiang Hospital, Southern Medical University, Guangzhou, and 'Department of Radiology, \\ Qingdao Central Hospital, Qingdao, China; ${ }^{\mathrm{d}}$ Department of Medicine, Renal Division, Emory University School of

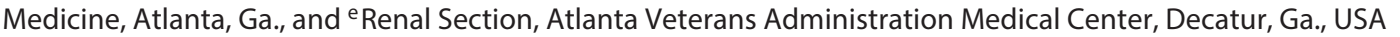

\section{Key Words}

WNK3 · Sodium chloride cotransporter · Lysosomal degradation · ERK 1/2 signaling

\begin{abstract}
Background: WNK kinase is a serine/threonine kinase that plays an important role in normal blood pressure homeostasis. WNK3 was previously found to enhance the activity of sodium chloride cotransporter (NCC) in Xenopus oocyte. However, the mechanism through which it works remains unclear. Methods: Using overexpression and siRNA knockdown techniques, the effects of WNK3 on NCC in both Cos-7 and mouse distal convoluted cells were analyzed by Western blot. Results: We found that WNK3 significantly increased NCC protein expression in a dose-dependent manner. NCC protein expression in Cos-7 cells was markedly decreased after $2 \mathrm{~h}$ treatment with protease inhibitor, cycloheximide $(\mathrm{CHX})$ in the NCC alone group, but was significantly decreased after $8 \mathrm{~h}$ treatment of $\mathrm{CHX}$ in the WNK3 + NCC group.
\end{abstract}

\section{KARGER}

(C) 2016 S. Karger AG, Basel

$1660-8151 / 16 / 1334-0287 \$ 39.50 / 0$

E-Mail karger@karger.com

www.karger.com/nef
WNK3 significantly increased NCC protein expression in both NCC alone and WNK3 + NCC groups regardless the overnight treatments of bafilomycin $\mathrm{A} 1$, a proton pump inhibitor, suggesting that WNK3-mediated increased NCC expression is not dependent on the lysosomal pathway. We further found that WNK3 group had a quicker NCC recovery than the control group using $\mathrm{CHX}$ pulse assay, suggesting that WNK3 increases NCC protein synthesis. WNK3 enhanced NCC protein level while reducing ERK 1/2 phosphorylation. In addition, knock-down of ERK $1 / 2$ expression reversed WNK3-mediated increase of NCC expression. Conclusion: These results suggest that WNK3 enhances NCC protein expression by increasing NCC synthesis via an ERK 1/2-dependent signaling pathway.

(c) 2016 S. Karger AG, Basel
Dr. Hui Cai

Renal Division, Emory University School of Medicine

1639 Pierce Drive, WMB Room 338

Atlanta, GA 30322 (USA)

E-Mail hcai3@emory.edu

Dr. Jieqiu Zhuang

Department of Nephrology, The Second Affiliated Hospital

Wenzhou Medical University

Wenzhou, Zhejiang 325000 (China)

E-Mail Jieqiuzhuang@ hotmail.com 


\section{Introduction}

WNK kinase is a subfamily of the serine/threonine kinases $[1,2]$. Human WNK consists of 4 isoforms, namely WNK1, WNK2, WNK3 and WNK4 $[1,2]$. The mutation of WNK1 and WNK4 cause pseudohypoaldosteronism type II (PHA II) featuring hypertension, hyperkalemia and metabolic acidosis [2]. WNK4 was shown to inhibit sodium chloride cotransporter (NCC) [3-5], whereas WNK3 was shown to activate NCC [6-8]. WNK3 is expressed throughout the nephron [6]. In addition, WNK3 was shown to stimulate sodium potassium chloride cotransporter and inhibit potassium chloride cotransporter and renal outer medullary potassium channel [6].

NCC is expressed in the renal distal convoluted tubule and is responsible for $5-7 \%$ of sodium reabsorption in the kidney [9]. Mutations in NCC cause Gitelman syndrome, an opposite phenotype of PHA II. NCC, which is regulated by either modulating its trafficking or intrinsic kinetics, has been reported to be modulated by angiotensin II [10], aldosterone [11, 12] or vasopressin [13]. WNK (1 or 4)-SPAK/OSR1 signaling plays an important role in NCC regulation [14-16]. A recent study showed that the interaction between WNK3 and SPAK is required for WNK3-mediated regulation of NCC [8]. In addition, WNK4 was found to inhibit NCC through an ERK 1/2-mediated signaling pathway [17]. While WNK3 has shown a powerful activation of NCC in vitro $[6,18]$, recent in vivo studies showed that WNK3 knockout (KO) mice exhibited only a small reduction of blood pressure during low salt diet, suggesting that WNK3 serves as a fine-tuning modulator in NCC regulation $[19,20]$. However, WNK1-SPAK signaling pathway is upregulated in WNK3 KO mice, which likely compensates for the loss of WNK3 activation of NCC [20]. A recent study reported that WNK3 prevented the Nedd4-2-mediated inhibition of NCC which is independent of both SPAK kinase and aldosterone-SGK1 pathway [21], suggesting that there might be an alternative pathway involving the WNK3mediated regulation of NCC in addition to WNK3-SPAK signaling pathway. However, the exact mechanism of WNK3-mediated NCC regulation remains to be clarified.

In the present study, we report that WNK3 increases NCC protein expression by enhancing NCC protein synthesis. WNK3 increases NCC expression while reducing ERK 1/2 phosphorylation, whereas knock-down of ERK $1 / 2$ expression reverses the WNK3-mediated increase of NCC expression, suggesting that the effect of WNK3 on NCC is mediated by a WNK3-ERK $1 / 2$ signaling pathway.

\section{Materials and Methods}

\section{Plasmids and Constructs}

Human WNK3 plasmid was kindly provided by Dr. Ji-Bin Peng at the University of Alabama at Birmingham. The human WNK3 was amplified by polymerase chain reaction using a human WNK3 plasmid as the template and matched to the human WNK3 sequence (GenBank Accession No.: NM_020922.4). The N-terminal Myc-tag was added to WT WNK3 construct and the WNK3 cDNA was subsequently subcloned into pCMV (Clontech, Mountain View, Calif., USA). Human NCC was amplified by PCR from a human kidney cDNA library and then subcloned into pCMV-HA vector (Clontech, Mountain View, Calif., USA) as previously described $[5,17]$. All constructs were confirmed by DNA sequencing. These constructs have been successfully expressed in an African green monkey kidney cell (Cos-7) as confirmed by Western blot analysis.

\section{Cell Culture and Transfection}

Cos-7 cells obtained from American Type Culture Collection (Manassas, Va., USA) were maintained in DMEM supplemented with $10 \%$ FCS, L-glutamine $(2 \mathrm{mM})$, penicillin $(100 \mathrm{U} / \mathrm{ml})$ and streptomycin $(100 \mu \mathrm{g} / \mathrm{ml})$. The mouse distal convoluted cells (mDCT cells) were obtained from Dr. Robert Hoover's laboratory and the cells were maintained in DMEM/F12 (1:1; Invitrogen) supplemented with penicillin $(100 \mathrm{U} / \mathrm{ml})$, streptomycin $(100 \mu \mathrm{g} /$ $\mathrm{ml}$ ) and 5\% fetal bovine serum. Lipofectamine 2000 (Invitrogen) was used for transfection of plasmids into mDCT cells according to the manufacturer's instructions. All other media and components were purchased from Life Technologies (Grand Island, N.Y., USA). Cells were transiently transfected using Lipofectamine 2000 (Life Technologies, Grand Island, N.Y., USA) according to the manufacturer's instructions. Forty-eight hours after transfecting cells with a series of doses of WNK3 plasmid, cells were lysed as previously described [5]. Cell lysates were subjected to SDS-PAGE and Western blot analysis. Lipofectamine RNAiMax (Invitrogen) was used for transfection of ERK $1 / 2$ siRNA into mDCT cells [17]. The synthetic siRNAs were obtained from Sigma. For transfection, $5 \mu \mathrm{l} 20 \mathrm{~mm}$ scramble siRNA or ERK 1/2 siRNA duplexes were added into $0.65 \mathrm{ml}$ Opti-MEM and mixed gently. Ten microliters Lipofectamine RNAiMAX were added into the diluted siRNAs solutions and mixed gently, incubated at room temperature for 15-20 min to allow siRNAs-liposome complex to form. The Opti-Mem solution containing siRNA liposome complex was added to a 3.35$\mathrm{ml} \mathrm{mDCT}$ cell suspension and the mixture was plated in $60-\mathrm{mm}$ dishes. Twenty-four hours after transfection, cells reached $60-70 \%$ confluences; additional plasmid will be transfected into the mDCT cells if needed.

\section{Western Blotting Analysis}

Cells were harvested and processed as described previously [5]. Briefly, cells transiently transfected with various DNA constructs or siRNA as indicated were lysed in lysis buffer containing $20 \mathrm{mM}$ Hepes, pH 7.5, 120 mM NaCl, 5.0 mM EDTA, 1.0\% Triton X-100, $0.5 \mathrm{mM}$ dithiothreitol, $1.0 \mathrm{mM}$ phenylmethylsulfonyl fluoride and complete protease inhibitor (Roche Diagnostics, Mannheim, Germany, 1 tablet per $50-\mathrm{ml}$ solution). The lysates were centrifuged at $6,000 \mathrm{~g}$ for $5 \mathrm{~min}$ to pellet the insoluble material, and the proteins from supernatant were quantified using a Pierce BCA Protein Assay kit (Pierce, Rockford, Ill., USA). After mixing in 
Fig. 1. WNK3 increases NCC protein expression in Cos-7 cells. Cos-7 cells were simultaneously co-transfected with $\mathrm{HA}$ tagged NCC $(1 \mu \mathrm{g})$ and different doses of Myc-tagged WNK3. Forty-eight hours after transfection cell lysates were subjected to SDS-PAGE and Western blot analysis. a A representative immunoblot for MycWNK3, HA-NCC and actin. b The bar graph representing the summarized data of total 4 experiments. The ratio of the HANCC over actin is presented as the percentage changes compared to the control group (WNK3 group at $0.5 \mu \mathrm{g}$ ). The WNK3 kinase increased NCC expression in a dosedependent manner. ${ }^{* *} \mathrm{p}<0.01, \mathrm{n}=4$, compared to the control group (WNK3 at $0.5 \mu \mathrm{g})$.

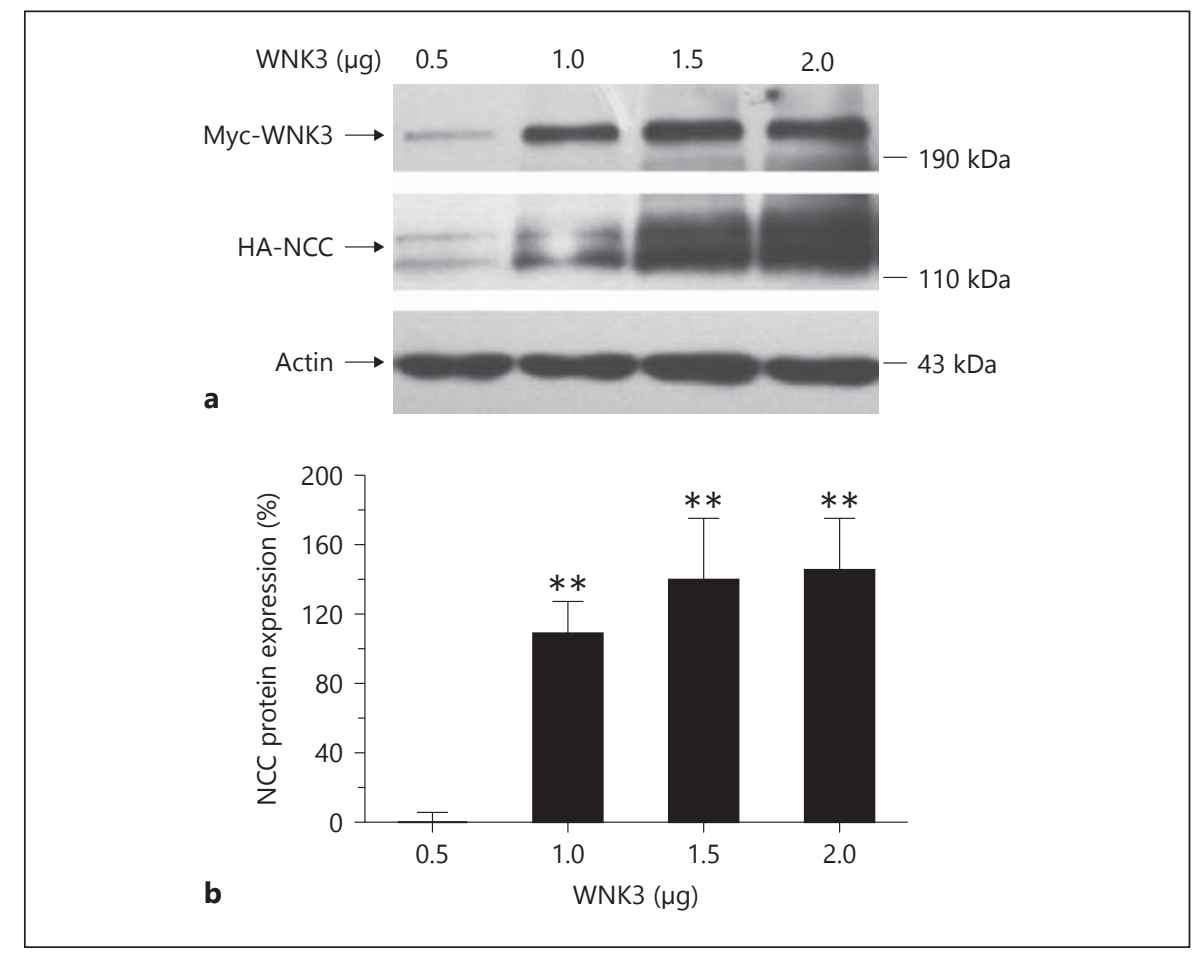

Laemmli buffer (Bio-Rad, Hercules, Calif., USA) and incubating at $37^{\circ} \mathrm{C}$ for $30 \mathrm{~min}$, the protein sample was separated by SDS-PAGE and electrophoretically transferred to polyvinylidene difluoride membranes (Amersham Biosciences, Piscataway, N.J., USA) for Western blot. Probing with specific antibodies and subsequent detection with ECL plus system (Amersham Biosciences) or Super signal (Pierce) were performed according to standard procedures as described previously [17]. Antibodies that were used include phospho-ERK 1/2 (p-ERK1/2) and total-ERK 1/2 (t-ERK1/2; Cell Signaling Technology, Boston, Mass., USA) and anti-actin (Millipore Corporation, Billerica, Mass., USA). NCC antibody is gift of Dr. Robert S. Hoover.

\section{Statistical Analysis}

The data are presented as the means \pm SE. Statistical significance was determined by Student's t test when 2 groups were compared and by one-way analysis of variance, followed by Bonferroni's post hoc tests when multiple groups were compared. We assigned significance at $\mathrm{p}<0.05$.

\section{Results}

\section{WNK3 Increased NCC Protein Expression}

After transfection with NCC $(1 \mu \mathrm{g})$ in combination with an increasing dose of WNK3 $(0.5,1.0,1.5$ and $2.0 \mu \mathrm{g})$ in Cos-7 cells, cell lysates were collected for Western blot analysis. As shown in figure 1, as WNK3 protein expression increases, the effect of WNK3 on NCC also gradu- ally increased, suggesting that WNK3 significantly enhanced NCC protein expressions in a dose-dependent manner $(108.8 \pm 18.7 \%$ at WNK3 $1 \mu \mathrm{g}, 141.5 \pm 33.0 \%$ at WNK3 $1.5 \mu \mathrm{g}$ and $146.0 \pm 27.9 \%$ at WNK3 $2.0 \mu \mathrm{g}$, compared to the control group with WNK3 at $0.5 \mu \mathrm{g}$; ${ }^{* *} \mathrm{p}<$ $0.01, \mathrm{n}=4)$. In addition, WNK3 also significantly increased the endogenous NCC expression in mDCT cells transfected with WNK3 (1.5 $\mu \mathrm{g})$ as shown in figure $2 \mathrm{a}$ and $b$.

\section{WNK3 Increases NCC Protein Expression by Stabilizing NCC Protein}

We have found that WNK3 significantly increased total NCC protein expression. It remains unclear whether protein translation is involved in the WNK3's stimulatory effect on NCC. Taking advantage of cycloheximide (CHX), a well-known protein synthesis inhibitor that inhibits new protein synthesis and with the presence of CHX treatment the existing synthesized proteins in the cells are gradually decreased over the time through the degradation pathway. Thus, we examined the effect of CHX treatment $(100 \mu \mathrm{g} / \mathrm{ml})$ on NCC in Cos-7 cells transfected with either NCC alone or NCC in combination with WNK3 $(1 \mu \mathrm{g})$. As seen in figure 3, NCC protein expression was significantly decreased after $2 \mathrm{~h}$ of CHX treatment in the NCC alone group 


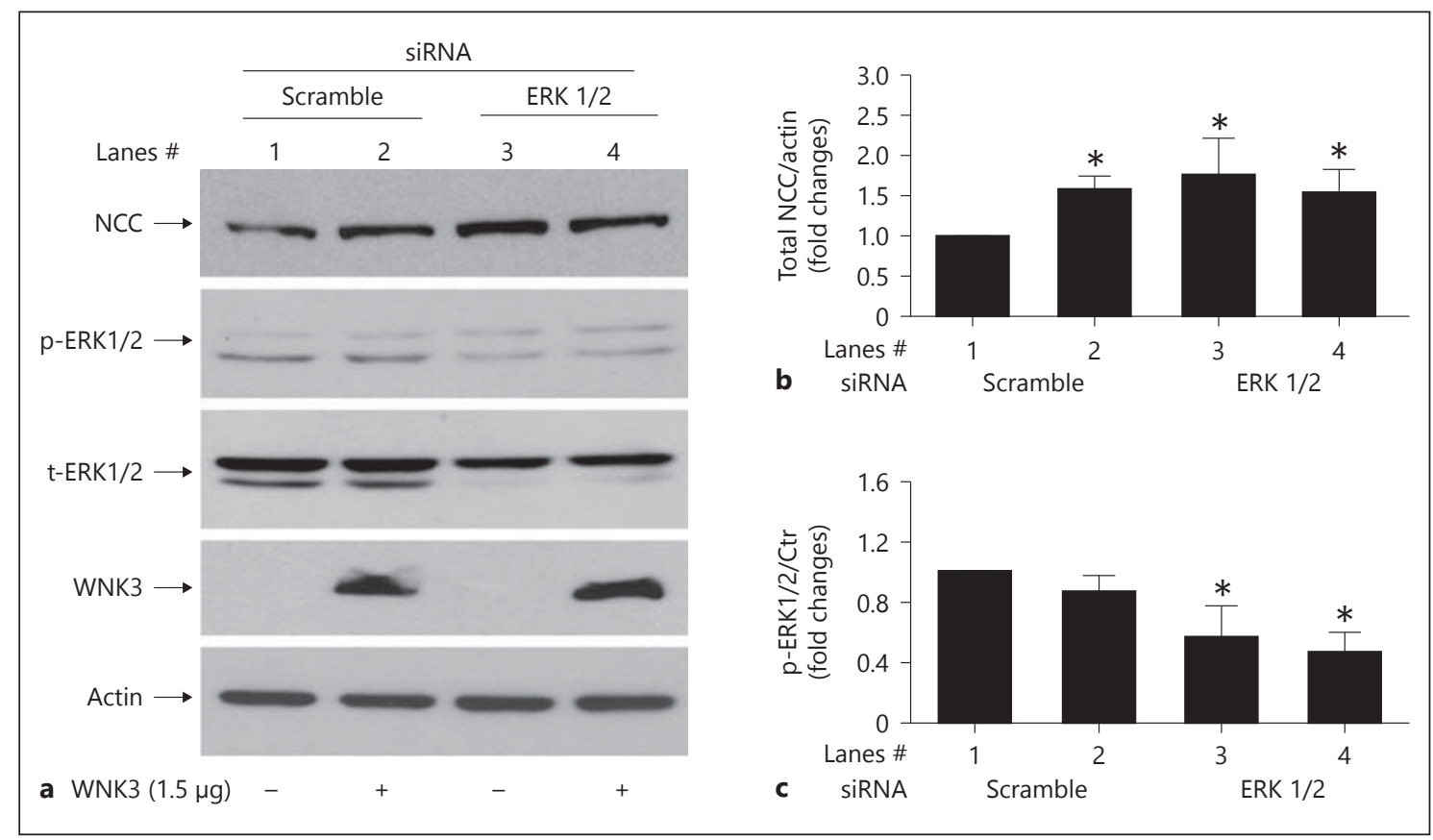

Fig. 2. Knock-down of ERK $1 / 2$ expression prevents the WNK3mediated increase of NCC in mDCT cells. The mDCT cells were transfected with or without siRNA ERK $1 / 2$ in combination with or without myc-WNK3 $(1.5 \mu \mathrm{g})$. Forty-eight hours after transfection, cell lysates were subjected to SDS-PAGE and Western blot analysis. Antibodies for tERK1/2, p-ERK1/2, myc (for WNK3), actin and NCC were used for Western blot analysis. a A representa-

(fig. 3a), whereas NCC protein expression started to markedly decrease after $8 \mathrm{~h}$ of $\mathrm{CHX}$ treatment in the WNK3 + NCC group (fig. 3b). These findings suggest that WNK3 stabilize the NCC protein by likely reducing the degradation of NCC.

\section{WNK3 Increased NCC by Likely Preventing Its}

Degradation Independent of a Lysosomal Pathway

Since we found that WNK3 protects NCC protein expression from degradation, we investigated which degradation pathway is involved in WNK3's regulation of NCC. Since NCC was found to target the lysosome for its degradation $[5,22,23]$, we examined the effect of bafilomycin A1 (Baf A1) on NCC protein expression. Baf A1 is a proton pump inhibitor that blocks the lysosomal degradation pathway. As shown in figure $4 \mathrm{a}$ and $\mathrm{b}$, WNK3 significantly increased NCC protein expression in both NCC alone and WNK3 + NCC groups regardless the overnight treatments of Baf A1. In the absence of Baf A1 treatment, WNK3 significantly increased NCC protein expression 2.34 folds (fig. 4a, lane 2) compared to the NCC alone group $(\mathrm{p}<0.01, \mathrm{n}=6$; fig. $4 \mathrm{a}$, lane 1$)$. After tive blot for NCC, WNK3, p-ERK1/2, t-ERK1/2 and actin. b A bar graph represents the summarized data of the ratio changes of NCC/actin from 4 independent experiments. $c$ A bar graph represents the summarized data of the ratio changes of $\mathrm{p}-\mathrm{ERK} 1 / 2 \mathrm{com}-$ pared to the basal p-ERK1/2 level (control-lane 1). ${ }^{*} \mathrm{p}<0.05$ compared to the control group (lane 1 ); $\mathrm{n}=4$.

the Cos-7 cells were pre-treated with Baf A1 $(0.5 \mu \mathrm{M})$, WNK3 also significantly enhanced NCC expression in the WNK3 + NCC group (fig. 4a, lane 4) compared to that in the NCC alone group with Baf A1 treatment (fig. 4a, lane 3). These data suggested that WNK3-mediated increased NCC expression is not mediated by degradation via a lysosomal pathway.

\section{WNK3 Increased NCC Protein Synthesis}

Since we found that WNK3-mediated upregulation of NCC is not involving the degradation via a lysosomal pathway, we tested whether or not WNK3 affects NCC protein synthesis. We performed the CHX pulse assay in the Cos-7 cells transfected with either NCC alone or WNK3 + NCC. Forty-eight hours after transfection, Cos7 cells were incubated with CHX $(100 \mu \mathrm{g} / \mathrm{ml})$ for $8 \mathrm{~h}$ and the mediums containing CHX were removed. Cells were harvested at 0, 30, 60, 120 and $240 \mathrm{~min}$. As shown in figure 5, Western blot analysis showed that NCC protein level was significantly increased 2 folds $(\mathrm{p}<0.05, \mathrm{n}=4)$ at $2 \mathrm{~h}$ after removal of CHX treatment in the WNK3 + NCC group, whereas NCC protein level was also signifi- 
Fig. 3. WNK3 increases NCC protein expression by reducing the degradation of NCC in Cos-7 cells. Cos-7 cells were transfected either HA-NCC alone $(1 \mu \mathrm{g})(\mathbf{a})$ or HA-NCC $(1 \mu \mathrm{g})$ and Myc-WNK3 $(1 \mu \mathrm{g})(\mathbf{b})$ as indicated. $\mathrm{CHX}$ chase assay was performed. a, b A representative blot for NCC alone group and NCC + WNK3 group, respectively. c A bar graph representing the summarized data of total 4 independent experiments. The ratio of the HA-NCC over actin is presented as the percentage changes compared to the control group (zero time point). Forty-eight hours after transfection, Cos-7 cells were incubated with $\mathrm{CHX}(100 \mu \mathrm{g} / \mathrm{ml})$ for different times $(0,1,2,4$ and $8 \mathrm{~h}$, respectively). Cells were harvested after incubations at different time points. Cell lysates were subjected to SDS-PAGE and Western blot analysis. Anti-HA and anti- $\beta$-actin antibodies were used for Western blot analysis. NCC protein levels were significantly reduced after $2 \mathrm{~h}$ incubation with $\mathrm{CHX}$ in the NCC alone group compared to NCC levels in the WNK3 + NCC group which were remarkably reduced after $8 \mathrm{~h}$ incubation with CHX, suggesting that WNK3 increases NCC protein by reducing the degradation of NCC. ${ }^{*} \mathrm{p}<0.05, \mathrm{n}=4$, compared to the control group (vector + NCC group) in the corresponding time point.

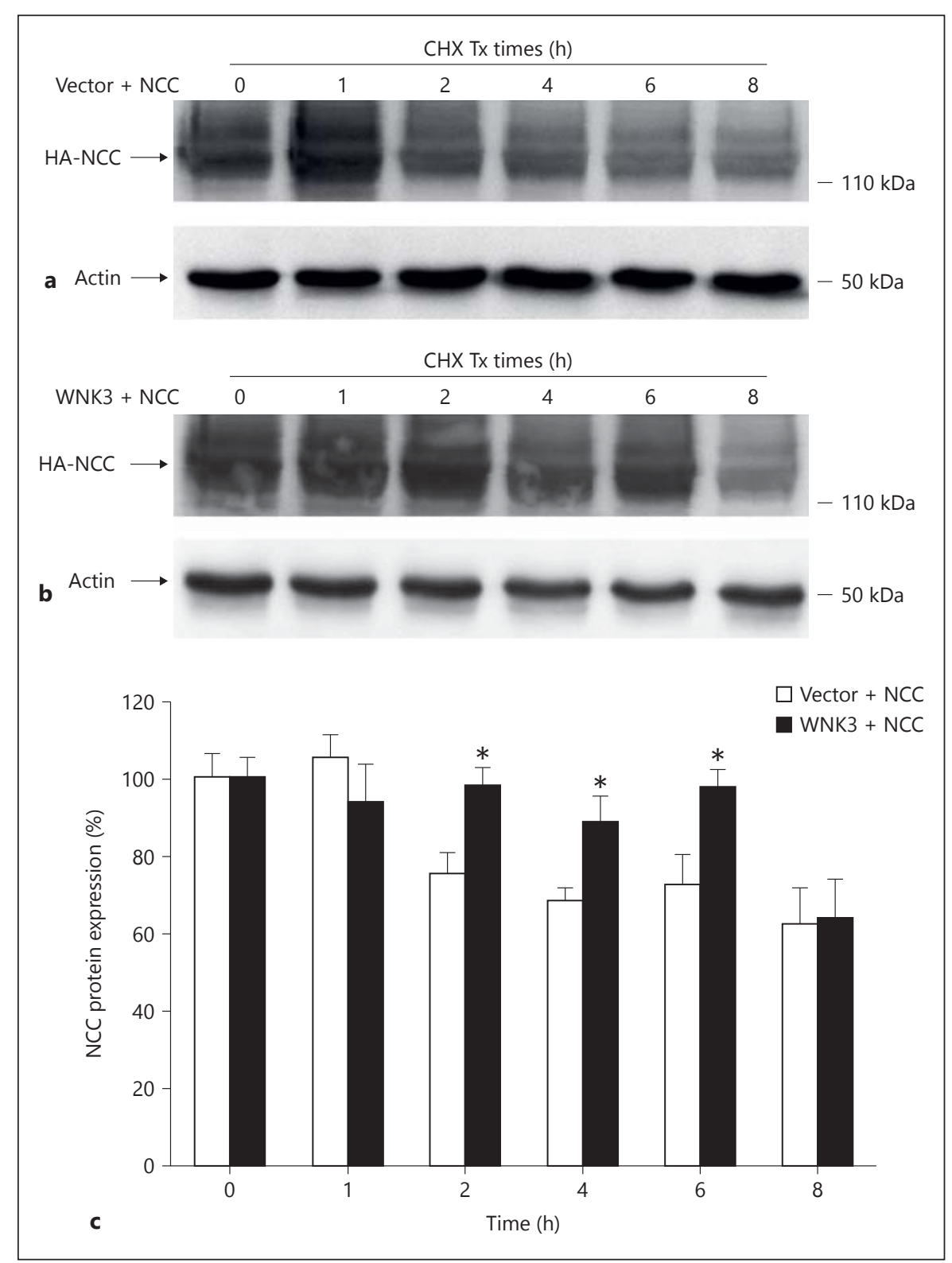

cantly increased $(\mathrm{p}<0.01, \mathrm{n}=4)$ at $4 \mathrm{~h}$ after removal of CHX treatment in NCC alone group, suggesting that WNK3 increases NCC protein synthesis.

\section{WNK3 Increases NCC Protein Expression While \\ Reducing ERK 1/2 Phosphorylation}

A previous study has shown that WNK3-SPAK interaction is required for the modulation of NCC [8]. A recent study showed that phosphorylation of SPAK, OSR1 and NCC in WNK3 KO mice were not changed [19]. We previously showed that WNK4 modulates NCC through an ERK 1/2 signaling pathway [17]. To explore the mecha- nism through which WNK3 affects NCC through modulating ERK 1/2 signaling pathway, we investigated the effect of WNK3 on ERK 1/2 phosphorylation. As shown in figure 6, WNK3 increased NCC protein expression, while reducing ERK 1/2 phosphorylation in a dose-dependent manner. These findings suggested that WNK3 increases NCC via modulating the ERK $1 / 2$ signaling pathway.

\section{Knock-Down of ERK 1/2 Expression Reverses the}

WNK3-Mediated Increases of NCC Expression

To confirm whether WNK3 upregulates NCC through ERK $1 / 2$ signaling pathway, we have performed the 
siRNA knockdown experiments. The mDCT cells were transfected with or without siRNA ERK $1 / 2$ in combination with or without WNK3 transfection, and endogenous NCC expressions were analyzed by Western blot. As shown in figure 2, overexpressing WNK3 alone significantly increased the endogenous NCC protein expression in mDCT cells compared to that in $\mathrm{mDCT}$ cells without WNK3. Knock-down of ERK 1/2 expression itself also increased NCC protein expression. However, knock-down of ERK $1 / 2$ expression prevented the
WNK3-mediated enhancement of NCC expression. These data suggest that WNK3 affects NCC via ERK 1/2 signaling pathway.

\section{Discussion}

WNK3 has been found to increase NCC activity and its protein expression [6]. WNK3 stimulates NCC activity likely through a SPAK signaling pathway [8]. In this

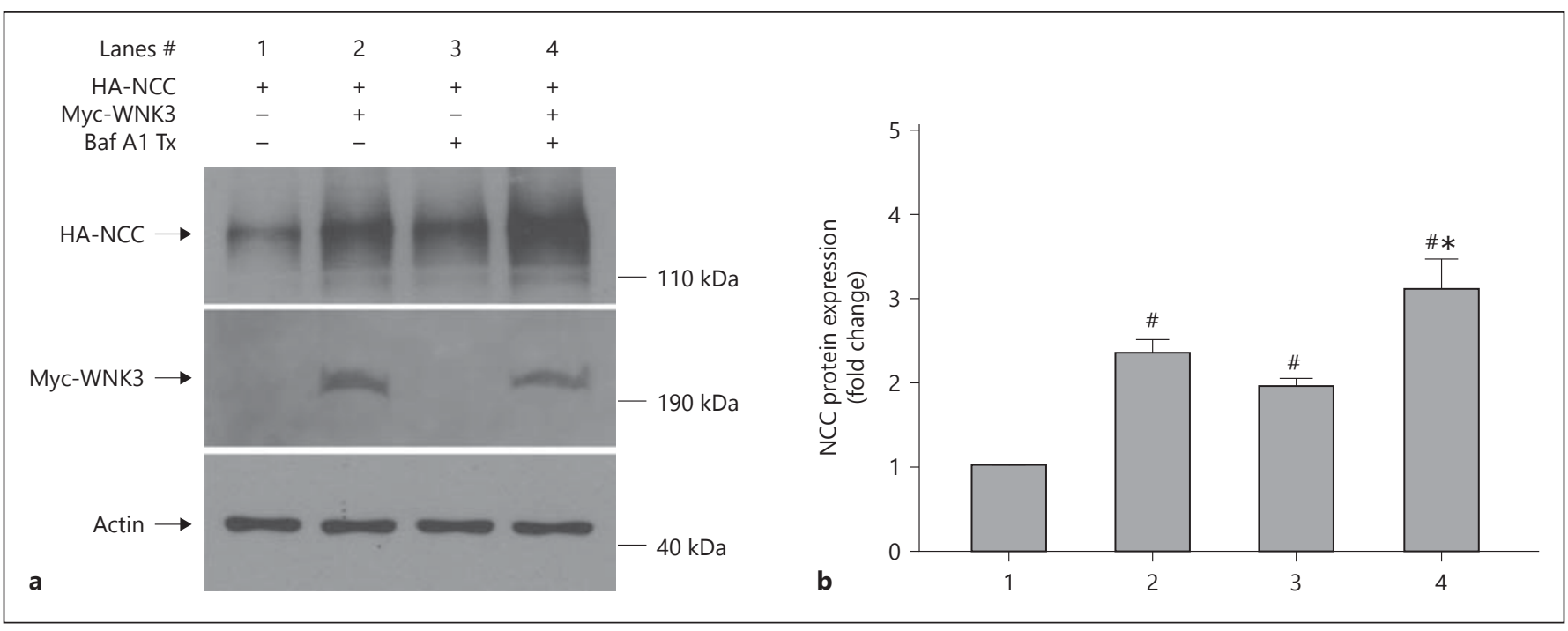

Fig. 4. WNK3 increases NCC protein expression independent of a lysosome-mediated degradation pathway. Cos-7 cells were cotransfected HA-NCC $(1 \mu \mathrm{g})$ with or without Myc-WNK3 $(1 \mu \mathrm{g})$. Thirty-six hours after transfection, the cells were treated with either Baf A1 $(0.5 \mu \mathrm{M})$ in Baf A1-treated groups (lanes 3 and 4) or DMSO vehicle in control groups (lanes 1 and 2 ) for $12 \mathrm{~h}$ before they were harvested for cell lysates. Cell lysates were subjected to SDS-PAGE and Western blot analysis. a A representative blot for NCC, WNK3 or actin. $\mathbf{b}$ Bar graph represents the summarized data for 6 independent experiments. WNK3 significantly increased NCC protein expression (lane 2) compared to the control NCC alone group (lane 1). Baf A1 treatment significantly increased

Fig. 5. WNK3 increases NCC protein synthesis in Cos-7 cells. Cos7 cells were transfected either HA-NCC alone $(2 \mu \mathrm{g})(\mathbf{a})$ or HANCC $(2 \mu \mathrm{g})$ and Myc-WNK3 (1.5 $\mu \mathrm{g})(\mathbf{b})$ as indicated. CHX pulse assay was performed. Forty-eight hours after transfection, Cos-7 cells were incubated with CHX $(100 \mu \mathrm{g} / \mathrm{ml})$ for $8 \mathrm{~h}$. CHX was removed by exchange the cell medium after $8 \mathrm{~h}$ of incubation. Cells were then harvested at different time points as indicated. Cell lysates were subjected to SDS-PAGE and Western blot analysis. a, b A representative blot for NCC alone group and NCC + WNK3 group, respectively. c, $\mathbf{d} \mathrm{A}$ bar graph representing the summarized
NCC protein expression in the NCC alone group (lane 3) compared to that in the NCC alone group without Baf A1 treatment (lane 1). WNK3 also significantly enhanced NCC protein expression in the Baf A1-treated WNK3 + NCC group (lane 4) compared to these either in the WNK3 + NCC group without Baf A1 treatment (lane 2) or in the NCC alone group with Baf A1 treatment (lane 3). These data showed that WNK3 increases NCC protein expression regardless of the treatments of Baf A1, suggesting that WNK3-mediated increase in NCC protein expression is independent of a lysosome-mediated degradation pathway. ${ }^{\#} \mathrm{p}<0.01 \mathrm{com}-$ pared to lane $1 ;{ }^{*} \mathrm{p}<0.05$ compared to either lane 2 or $3 ; \mathrm{n}=6$. data of total 4 independent experiments. The ratio of the HA-NCC over actin is presented as the percentage changes compared to the control group (zero time point). Anti-HA and anti- $\beta$-actin antibodies were used for Western blot analysis. NCC protein level was significantly increased at $2 \mathrm{~h}$ after removal of $\mathrm{CHX}$ treatment in the WNK3 + NCC group, whereas NCC protein level was significantly increased at $4 \mathrm{~h}$ after removal of CHX treatment in NCC alone group, suggesting that WNK3 increases NCC protein synthesis. ${ }^{*} \mathrm{p}<0.05,{ }^{* *} \mathrm{p}<0.01, \mathrm{n}=4$, compared to the control group at the respective zero time points.

(For figure see next page.) 

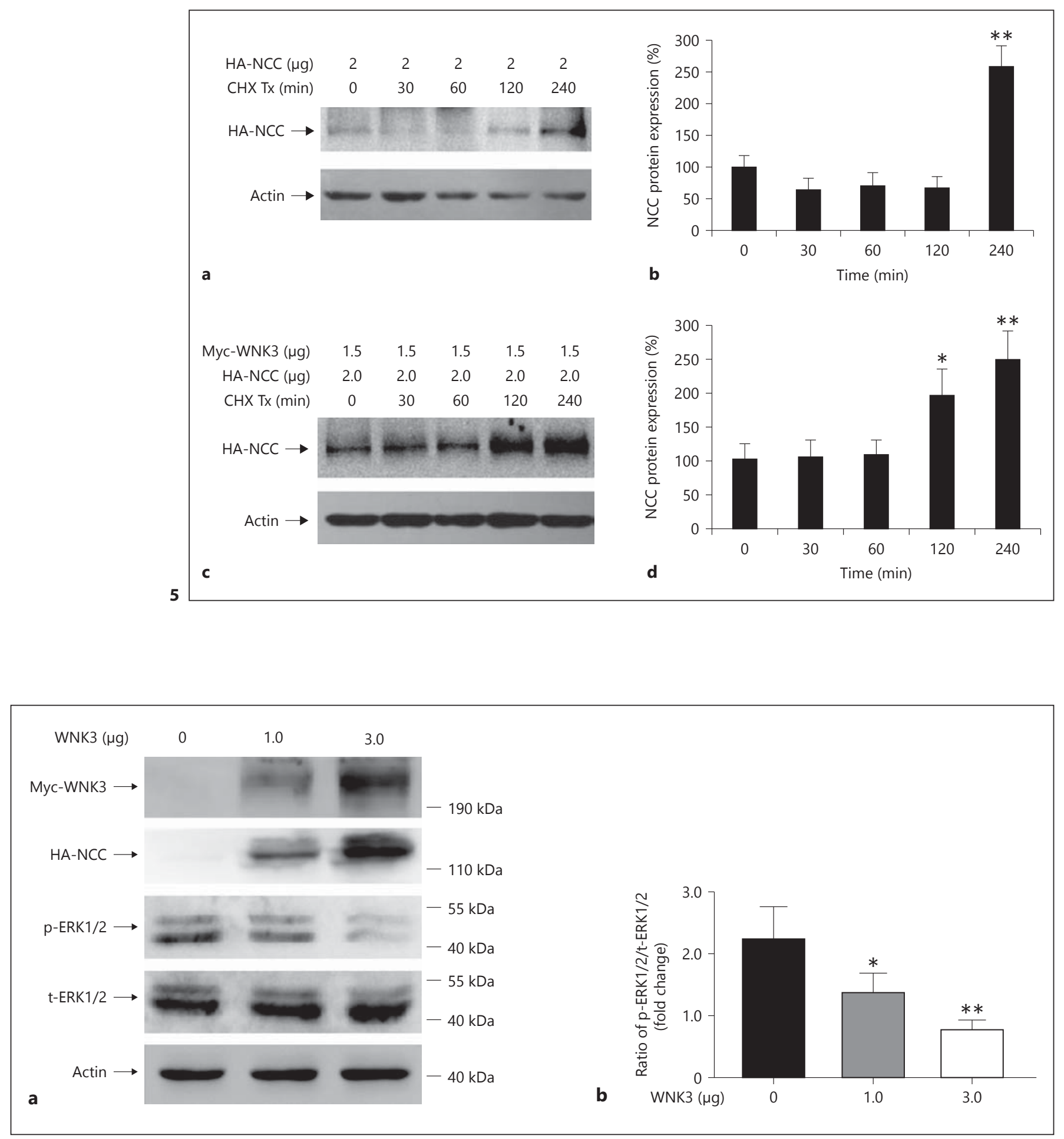

Fig. 6. WNK3 increases NCC protein expressions by reducing ERK $1 / 2$ phosphorylation. Cos-7 cells were transfected with HA-NCC and an increasing dose of Myc-WNK3. Forty-eight hours after transfection, cell lysates were subjected to SDS-PAGE and Western blot analysis. Antibodies for HA (for NCC), tERK1/2, pERK1/2, and myc (for WNK3) were used for Western blot analysis. a A representative blot for $\mathrm{WNK} 3, \mathrm{NCC}, \mathrm{p}-\mathrm{ERK} 1 / 2$ and total $\mathrm{t}$ -
ERK1/2. b A bar graph represents the summarized data of the ratio of p-ERK1/2/t-ERK1/2 from 4 independent experiments. Data showed that WNK3 significantly increases NCC while reducing ERK $1 / 2$ phosphorylation in a dose-dependent manner. ${ }^{*} \mathrm{p}<0.05$ compared to the control group $(0 \mu \mathrm{g}$ WNK 3$),{ }^{* *} \mathrm{p}<0.01$ compared to the control group $(0 \mu \mathrm{g} \mathrm{WNK} 3) ; \mathrm{n}=4$. 
study, we found that WNK3 increased NCC protein expression by stabilizing NCC, but not mediated degradation via a lysosomal pathway. WNK3 likely increases NCC synthesis. WNK3 enhances NCC via modulating the ERK $1 / 2$ signaling pathway.

WNK kinase is a subfamily of serine/threonine kinases [24]. WNK kinase plays an important role in regulating NCC $[25,26]$. WNK4 and WNK3 were found to modulate NCC activity, but in an opposite direction $[3-6,18]$. A previous study found that the amino terminus of WNK3 and WNK4 confers their critical difference in the regulation of NCC [7]. WNK3 was also found to be a chloride sensitive or responsive kinase [27]. While both WNK3 and WNK4 modulate NCC activity through activating the SPAK signaling pathway, their effects on NCC activity appear to be different $[8,14,15,26,28]$. The exact explanation of why WNK3 and WNK4 have different effects on NCC remains elusive. Our previous studies showed that WNK4 inhibits NCC by enhancing NCC degradation through a lysosomal pathway $[5,22]$. In the present study (fig. 4), we found that WNK3 significantly increased NCC protein expression. WNK3 significantly increased NCC protein expression in both NCC alone and WNK3 + NCC groups regardless the overnight treatments of Baf $\mathrm{A} 1$. These data suggested that WNK3 increases NCC expression independent of the lysosome-mediated degradation pathway. There is the other possibility that WNK3 could affect NCC synthesis. We have done CHX pulse assay to explore this possibility. We found that WNK3 dose increases NCC synthesis as shown in figure 5.

Previous studies reported that the interaction between WNK3 and SPAK is required for NCC regulation, suggesting that WNK3 modulates NCC through a SPAK signaling pathway $[6,8]$. However, a recent study demonstrated that WNK3 activates NCC activity by preventing the Nedd4-2-mediated inhibitory effect on NCC [21], an effect independent of both the activation of the WNK3SPAK signaling pathway [8] and the aldosterone-mediated SGK1 pathway [29]. Previous studies demonstrated that the ERK $1 / 2$ signaling pathway modulates NCC activity $[17,30]$. Our previous study also showed that WNK4 modulates NCC via ERK $1 / 2$ signaling [17]. Enhancing ERK 1/2 phosphorylation was found to lead to increasing NCC ubiquitination and degradation [31]. In the present study, we found that WNK3 increased NCC protein expression while reducing ERK $1 / 2$ phosphorylation, whereas knock-down of ERK 1/2 expression prevented the WNK3-mediated enhancement of NCC expression in mDCT cells, suggesting that WNK3 modulates NCC via an ERK 1/2 signaling pathway.
Recent animal studies reported that WNK3 $\mathrm{KO}$ mice exhibit a small, but significant reduction in blood pressure during low-salt diet challenge while showing a compensatory increase in WNK1 expression $[19,20]$. WNK1 has been shown to stimulate NCC activity in a WNK4independent, but SPAK-dependent mechanism [26]. The limited phenotype of low blood pressure in WNK3 KO mice support the notion that the decrease in NCC abundance results from loss of WNK3 expression and is offset by compensatory overexpression of $\mathrm{WNK} 1$, indicating a complex WNK signaling network involving physiological regulation of NCC in vivo. Clinically the mutation of WNK1 and WNK4 cause PHA II, featuring hypertension, hyperkalemia and metabolic acidosis [2]. Although WNK3 itself is not responsible for the phenotypic features of PHA II, the complexity of WNK signaling pathway indicates that WNK kinases including WNK3 play an important role in maintaining electrolyte homeostasis, particularly in regulation of sodium balance and blood pressure regulation.

In summary, our present study demonstrates that WNK3 increases NCC protein expression by enhancing NCC protein synthesis through an ERK $1 / 2$ signalingmediated mechanism, providing an additional pathway for WNK3-mediated NCC regulation.

\section{Acknowledgments}

This work is supported by the Department of Veteran Affairs MERIT Award 5I01BX000994 (H.C.), the National Institutes of Health R01 DK-085097 (R.S.H.), the National Natural Science Foundation of China \#81200513 (D.W.), Qianjiang Talents Project of Technology Office of Zhejiang Province \#2011R10049 (D.W.), and Wenzhou Science and Technology Bureau \#H20110014 (D.W.); National Natural Science Foundation of China \#81170709 (J.Z.), Zhejiang Provincial Natural Science Foundation of China \#Y2080291 (J.Z.), and Wenzhou City Science and Technology Cooperation Program H20090022 (J.Z.); Zhejiang Provincial Natural Science Foundation of China \#Y2090529 (D.G.) and Wenzhou City Science and Technology Cooperation Program H20090071 (D.G.). We thank Dr. Janet Klein for her suggestions and critical reading of this manuscript.

\section{Disclosure Statement}

All the authors declared no conflict of interest.

\section{Statement of Ethics}

This study did not require informed consent nor review/approval by the appropriate ethics committee. 


\section{References}

$>1$ Xu B, English JM, Wilsbacher JL, Stippec S, 11 Chiga M, Rai T, Yang SS, Ohta A, Takizawa Goldsmith EJ, Cobb MH: WNK1, a novel mammalian serine/threonine protein kinase lacking the catalytic lysine in subdomain II. J Biol Chem 2000;275:16795-16801.

$\checkmark 2$ Wilson FH, Disse-Nicodème S, Choate KA, Ishikawa K, Nelson-Williams C, Desitter I, Gunel M, Milford DV, Lipkin GW, Achard JM, Feely MP, Dussol B, Berland Y, Unwin RJ, Mayan H, Simon DB, Farfel Z, Jeunemaitre X, Lifton RP: Human hypertension caused by mutations in WNK kinases. Science 2001;293: 1107-1112.

$>3$ Wilson FH, Kahle KT, Sabath E, Lalioti MD, Rapson AK, Hoover RS, Hebert SC, Gamba G, Lifton RP: Molecular pathogenesis of inherited hypertension with hyperkalemia: the $\mathrm{Na}-\mathrm{Cl}$ cotransporter is inhibited by wild-type but not mutant WNK4. Proc Natl Acad Sci U S A 2003;100:680-684.

4 Yang CL, Zhu X, Wang Z, Subramanya AR, Ellison DH: Mechanisms of WNK1 and WNK4 interaction in the regulation of thiazide-sensitive $\mathrm{NaCl}$ cotransport. J Clin Invest 2005;115:1379-1387.

5 Cai H, Cebotaru V, Wang YH, Zhang XM, Cebotaru L, Guggino SE, Guggino WB: WNK4 kinase regulates surface expression of the human sodium chloride cotransporter in mammalian cells. Kidney Int 2006;69:21622170.

$\checkmark 6$ Rinehart J, Kahle KT, de Los Heros P, Vazquez N, Meade P, Wilson FH, Hebert SC, Gimenez I, Gamba G, Lifton RP: WNK3 kinase is a positive regulator of NKCC2 and NCC, renal cation-Cl- cotransporters required for normal blood pressure homeostasis. Proc Natl Acad Sci U S A 2005;102: 16777-16782.

7 San-Cristobal P, Ponce-Coria J, Vazquez N, Bobadilla NA, Gamba G: WNK3 and WNK4 amino-terminal domain defines their effect on the renal $\mathrm{Na}+-\mathrm{Cl}-$ cotransporter. Am J Physiol Renal Physiol 2008;295:F1199F1206.

$>8$ Pacheco-Alvarez D, Vázquez N, CastañedaBueno M, de-Los-Heros P, Cortes-González C, Moreno E, Meade P, Bobadilla NA, Gamba $\mathrm{G}$ : WNK3-SPAK interaction is required for the modulation of NCC and other members of the SLC12 family. Cell Physiol Biochem 2012;29:291-302.

>9 Gamba G: Molecular physiology and pathophysiology of electroneutral cation-chloride cotransporters. Physiol Rev 2005;85:423493.

-10 Castañeda-Bueno M, Cervantes-Pérez LG, Vázquez N, Uribe N, Kantesaria S, Morla L, Bobadilla NA, Doucet A, Alessi DR, Gamba $\mathrm{G}$ : Activation of the renal $\mathrm{Na}+\mathrm{Cl}$ - cotransporter by angiotensin II is a WNK4-dependent process. Proc Natl Acad Sci U S A 2012; 109:7929-7934.
T, Sasaki S, Uchida S: Dietary salt regulates the phosphorylation of OSR1/SPAK kinases and the sodium chloride cotransporter through aldosterone. Kidney Int 2008;74: 1403-1409.

12 Lai L, Feng X, Liu D, Chen J, Zhang Y, Niu B, $\mathrm{Gu}$ Y, Cai H: Dietary salt modulates the sodium chloride cotransporter expression likely through an aldosterone-mediated WNK4ERK1/2 signaling pathway. Pflugers Arch 2012;463:477-485.

13 Pedersen NB, Hofmeister MV, Rosenbaek LL, Nielsen J, Fenton RA: Vasopressin induces phosphorylation of the thiazide-sensitive sodium chloride cotransporter in the distal convoluted tubule. Kidney Int 2010;78:160169.

14 Richardson C, Rafiqi FH, Karlsson HK, Moleleki N, Vandewalle A, Campbell DG, Morrice NA, Alessi DR: Activation of the thiazide-sensitive $\mathrm{Na}+\mathrm{Cl}$ - cotransporter by the WNK-regulated kinases SPAK and OSR1. J Cell Sci 2008;121(pt 5):675-684.

15 Richardson C, Alessi DR: The regulation of salt transport and blood pressure by the WNK-SPAK/OSR1 signalling pathway. J Cell Sci 2008;121(pt 20):3293-3304.

16 Grimm PR, Taneja TK, Liu J, Coleman R, Chen YY, Delpire E, Wade JB, Welling PA: SPAK isoforms and OSR1 regulate sodiumchloride co-transporters in a nephron-specific manner. J Biol Chem 2012;287:3767337690.

17 Zhou B, Wang D, Feng X, Zhang Y, Wang Y, Zhuang J, Zhang X, Chen G, Delpire E, Gu D, Cai H: WNK4 inhibits NCC protein expression through MAPK ERK1/2 signaling pathway. Am J Physiol Renal Physiol 2012;302: F533-F539.

18 Cruz-Rangel S, Melo Z, Vázquez N, Meade P, Bobadilla NA, Pasantes-Morales H, Gamba G, Mercado A: Similar effects of all WNK3 variants on SLC12 cotransporters. Am J Physiol Cell Physiol 2011;301:C601-C608.

19 Oi K, Sohara E, Rai T, Misawa M, Chiga M, Alessi DR, Sasaki S, Uchida S: A minor role of WNK3 in regulating phosphorylation of renal NKCC2 and NCC co-transporters in vivo. Biol Open 2012;1:120-127.

20 Mederle K, Mutig K, Paliege A, Carota I, Bachmann S, Castrop H, Oppermann M: Loss of WNK3 is compensated for by the WNK1/ SPAK axis in the kidney of the mouse. Am J Physiol Renal Physiol 2013;304:F1198F1209.

21 Lagnaz D, Arroyo JP, Chávez-Canales M, Vázquez N, Rizzo F, Spirlí A, Debonneville A, Staub O, Gamba G: WNK3 abrogates the NEDD4-2-mediated inhibition of the renal $\mathrm{Na}+-\mathrm{Cl}-$ cotransporter. Am J Physiol Renal Physiol 2014;307:F275-F286.
22 Zhou B, Zhuang J, Gu D, Wang H, Cebotaru L, Guggino WB, Cai H: WNK4 enhances the degradation of NCC through a sortilin-mediated lysosomal pathway. J Am Soc Nephrol 2010;21:82-92.

-23 Subramanya AR, Liu J, Ellison DH, Wade JB, Welling PA: WNK4 diverts the thiazide-sensitive $\mathrm{NaCl}$ cotransporter to the lysosome and stimulates AP-3 interaction. J Biol Chem 2009;284:18471-18480.

24 Kahle KT, Ring AM, Lifton RP: Molecular physiology of the WNK kinases. Annu Rev Physiol 2008;70:329-355.

25 Glover M, O’shaughnessy KM: Molecular insights from dysregulation of the thiazide-sensitive WNK/SPAK/NCC pathway in the kidney: Gordon syndrome and thiazide-induced hyponatraemia. Clin Exp Pharmacol Physiol 2013;40:876-884.

26 Chávez-Canales M, Zhang C, Soukaseum C, Moreno E, Pacheco-Alvarez D, Vidal-Petiot E, Castañeda-Bueno M, Vázquez N, Rojas-Vega L, Meermeier NP, Rogers S, Jeunemaitre X, Yang CL, Ellison DH, Gamba G, Hadchouel J: WNK-SPAK-NCC cascade revisited: WNK1 stimulates the activity of the $\mathrm{Na}-\mathrm{Cl}$ cotransporter via SPAK, an effect antagonized by WNK4. Hypertension 2014;64:1047-1053.

27 Ponce-Coria J, San-Cristobal P, Kahle KT, Vazquez N, Pacheco-Alvarez D, de Los Heros P, Juárez P, Muñoz E, Michel G, Bobadilla NA, Gimenez I, Lifton RP, Hebert SC, Gamba G: Regulation of NKCC2 by a chloride-sensing mechanism involving the WNK3 and SPAK kinases. Proc Natl Acad Sci U S A 2008; 105:8458-8463.

28 San-Cristobal P, Pacheco-Alvarez D, Richardson C, Ring AM, Vazquez N, Rafiqi FH, Chari D, Kahle KT, Leng Q, Bobadilla NA, Hebert SC, Alessi DR, Lifton RP, Gamba G: Angiotensin II signaling increases activity of the renal $\mathrm{Na}-\mathrm{Cl}$ cotransporter through a WNK4-SPAK-dependent pathway. Proc Natl Acad Sci U S A 2009;106:4384-4389.

-29 Arroyo JP, Lagnaz D, Ronzaud C, Vázquez N, Ko BS, Moddes L, Ruffieux-Daidié D, Hausel P, Koesters R, Yang B, Stokes JB, Hoover RS, Gamba G, Staub O: Nedd4-2 modulates renal $\mathrm{Na}+-\mathrm{Cl}$ - cotransporter via the aldosteroneSGK1-Nedd4-2 pathway. J Am Soc Nephrol 2011;22:1707-1719.

30 Ko B, Cooke LL, Hoover RS: Parathyroid hormone $(\mathrm{PTH})$ regulates the sodium chloride cotransporter via Ras guanyl releasing protein 1 (Ras-GRP1) and extracellular signal-regulated kinase (ERK)1/2 mitogen-activated protein kinase (MAPK) pathway. Transl Res 2011;158:282-289.

31 Ko B, Kamsteeg EJ, Cooke LL, Moddes LN, Deen PM, Hoover RS: RasGRP1 stimulation enhances ubiquitination and endocytosis of the sodium-chloride cotransporter. Am J Physiol Renal Physiol 2010;299:F300-F309.
WNK3 Activates NCC via ERK 1/2

Signaling Pathway
Nephron 2016;133:287-295 DOI: $10.1159 / 000447717$ 